\title{
ECG-based Feature Tracking in Atrial Tachyarrhythmias
}

\author{
M Stridh ${ }^{1}$, L Sörnmo ${ }^{1}$, S B Olsson ${ }^{2}$ \\ ${ }^{1}$ Dept. of Electroscience, ${ }^{2}$ Dept. of Cardiology, Lund University, Sweden
}

\begin{abstract}
A new method for extraction of general features in ECGs with atrial tachyarrhythmias is presented. The method is based on our recent method for atrial signal characterization which sequentially decomposes a timefrequency distribution into a set of parameters. In addition to rate and amplitude, the method tracks information on regularity, waveform, and structure of the atrial signal. The proposed method includes a feature tracker which continuously tracks the structure of the harmonic spectral pattern in order to determine which of a set of archetype waveforms that is best matched. The method also includes a trend detector, which detects significant longterm changes in the time series of frequency estimates. The results, illustrated by signals obtained during different interventions and a test signal, show that the algorithms can discriminate between different types of atrial rhythms.
\end{abstract}

\section{Introduction}

The exact mechanisms of atrial fibrillation and its relation to other atrial arrhythmias remain uncertain. While a large number of measures relate amplitudes, durations and morphology in the ECG to various mechanisms of ventricular arrhythmias, only very basic measures such as overall amplitude and rate have been used for atrial arrhythmias. In the ECG, three main atrial tachyarrhythmias may be discerned: focal atrial tachycardia $(2.5-3.3 \mathrm{~Hz}$, regular), atrial flutter $(3.3-5.7 \mathrm{~Hz}$, regular, typical atrial flutter is sawtooth-shaped), and atrial fibrillation (4-9 Hz, irregular). Thus, rate and regularity should, based on the above definitions, be sufficient for discrimination of these arrhythmias. However, recent techniques have revealed large, rapid variations in repetition rate as well as both regular and irregular intervals occurring within the same recording [1], possibly indicating that the dynamics of the atrial signal could play a role when classifying atrial tachyarrhythmias.

In order to perform a detailed analysis of atrial tachyarrhythmias the atrial signal needs to be extracted from the ECG signal. A major difficulty has been to extract clean atrial signals from the ECG because of the dominant ventricular activity. During recent years, several approaches for atrial signal extraction (or QRST cancellation) have been suggested, see e.g. [2], [3], [4]. Atrial signal characterization, based on the residual ECG following QRST cancellation, has been done by tracking the dynamics in the atrial signal properties [1], [5]. In a recent approach, VCG loops of individual f-waves were studied during typical atrial flutter before and after ablation [6].

\section{Methods}

In this paper, we propose a system for atrial signal analysis which consists of atrial signal extraction [4], detailed atrial signal characterization [5], and two new analysis steps for feature tracking and frequency trend detection which are the focus in this study. The signal characterization method parameterizes a time-frequency distribution of the atrial signal into an exponentially updated spectral profile, describing the present harmonic pattern, and a set of trends describing, e.g., frequency and amplitude variations of the signal [5]. The main idea is to decompose the time-frequency distribution of the atrial signal such that each spectrum can be represented by a frequency-shifted and amplitude-scaled version of the spectral profile. The method operates sequentially in order to allow for adaptation of the spectral profile.

\subsection{The atrial rhythm feature tracker (ARFT)}

The main objective of the feature tracker is to translate the signal parameters provided by the characterization stage into a set of general rhythm features. The analysis is based on nonoverlapping segments of $N_{1}$ samples of the second-to-second resolution parameters from the atrial signal characterizer. Three indicators describing different general features of the atrial signals have been defined: the atrial tachyarrhythmia indicator (ATAI) is the most important indicator and keeps track of the local structure of the signal and indicates whether an atrial tachyarrhythmia is present or not. The regularity indicator quantifies the regularity of the atrial repetition rate. Finally, the waveform indicator translates the spectral amplitudes of the harmonics into three "archetype" waveforms. 
In addition to these indicators, averages of the frequency and amplitude time series are calculated. The ATAI is described in Sec. 2.1.1 and the regularity and waveform indicators are described in Secs. 2.1.2 and 2.1.3, respectively.

\subsubsection{The atrial tachyarrhythmia indicator}

The ATAI is based on signal structure and local noise.

Signal structure: The spectral profile is used to detect a valid atrial tachyarrhythmia signal structure. The spectral profile at the end of each segment of $N_{1}$ seconds is used since it reflects the over-all signal structure of the entire segment. Three different properties of the spectral profile are employed for detecting a valid signal structure. Empirically, we have found that atrial tachyarrhythmic ECGs have an harmonic structure with decaying amplitudes. The following measures reflect information on such a structure.

1. The signal-to-noise ratio (SNR) of the spectral profile is defined as the the ratio between the mean of the fundamental and the first harmonic amplitudes, and the background noise level where the noise level is the amplitude at the position halfway between the fundamental and first harmonic positions.

2. The second peak position (SPP) of the spectral profile measures the ratio between the distance (number of frequency points) between the positions of the fundamental (which by definition is the largest peak) and the second largest peak in the spectral profile and the distance between the positions of the fundamental and the first harmonic. The motivation for introducing the SPP is that signals without atrial activity above $2.5 \mathrm{~Hz}$, e.g., with $\mathrm{P}$ waves having a slower rate, often result in a ringing spectral profile. The second largest peak of such a ringing profile is, however, often very close to the largest peak and not at the expected position of the first harmonic.

3. The second peak amplitude (SPA) of the spectral profile is related to the SPP and measures the ratio between the fundamental amplitude and the amplitude of the second largest peak. The SPA is used to detect a too high first harmonic since the atrial signal characterization method in [5] is constructed for waveforms with a decaying harmonic spectrum.

Combining the above three parameters, the binary variable $z_{V S S}(l)(\mathrm{VSS}=$ valid signal structure) is set to one when the SNR is sufficiently high and neither SPP nor SPA indicates a ringing spectral profile. Since the spectral profile is updated exponentially, instantaneous changes or disturbances are not reflected by the spectral profile.

Local noise: In order to detect local noise (e.g., due to muscular activity or a poorly canceled QRS complex) or the onset of a structural change, the model error, $e(n)$ between the amplitude-scaled spectral profile and the present spectrum, is employed to set the binary variable local noise (LN). We suggest to threshold a sliding average of the model error for the purpose of finding local disturbances. The binary $z_{L N}(l)$ variable is set to one if $e(n)$ somewhere during the nonoverlapping segment, $l$, of $N_{1}$ samples exceeds $\alpha$ times the sliding average, $\bar{e}(l)$, over the last $N_{a}$ samples.

Depending on $z_{V S S}(l)$ and $z_{L N}(l)$, the ATAI is assigned one of the three labels:

$$
z_{A T A I}(l)= \begin{cases}\text { Arrh. present } & z_{V S S}(l) \& \overline{z_{L N}(l)} \\ \text { Local noise } & \frac{z_{V S S}(l) \& z_{L N}(l)}{z_{V S S}(l)}\end{cases}
$$

\subsubsection{The regularity indicator}

The regularity indicator is a very important indicator designed to discriminate flutter and tachycardia from fibrillation. The regularity indicator is based on the standard deviation, $\sigma(l)$, of the $N_{1}$ samples of the last block of the time series of frequency estimates. The standard deviation, $\sigma(l)$, is discretized into three levels of regularity:

$$
z_{R I}(l)= \begin{cases}\text { Regular } & \sigma(l)<r_{1} \\ \text { Irregular } & r_{1} \leq \sigma(l) \leq r_{2} \\ \text { Highly irregular } & \sigma(l)>r_{2}\end{cases}
$$

\subsubsection{The waveform indicator}

The waveform indicator reflects over-all morphology and is parameterized by using the spectral line model in which the fundamental and harmonic amplitudes are modeled as exponentially decaying [5]. The estimated exponential decay at time $n$ is denoted $\gamma(n)$. The exponential decay is discretized into the waveform indicator using the three levels:

$$
z_{W I}(l)= \begin{cases}\text { Pulse-shaped } & \gamma\left(l N_{1}\right)<w_{1} \\ \text { Sawtooth-like } & w_{1} \leq \gamma\left(l N_{1}\right) \leq w_{2} \\ \text { Fluctuating } & \gamma\left(l N_{1}\right)>w_{2}\end{cases}
$$

\subsection{The frequency trend detector (FTD)}

The FTD summarizes the frequency estimates in segments of $N_{2}\left(>N_{1}\right)$ samples into their average frequency and related variance, and detects relative changes in the time series of frequency estimates. The FTD is implemented such that it first searches for an initial frequency state, then detects deviations from this state, and successively defines new states. It is here assumed that the $N_{2}$ frequency estimates have a Gaussian distribution around an unknown mean with an unknown variance.

\section{ECG examples}

All signals were recorded using the standard 12-lead ECG. Only lead $V_{1}$ was subjected to classification and 
trend detection, although $V_{2}$ and $V_{3}$ were used for QRST cancellation. The signals were digitized at a sampling rate of $1 \mathrm{kHz}$ using equipment by Siemens-Elema AB, Sweden. The following recordings were used:

1. Sotalol intervention: 20 minute recording from a patient with atrial fibrillation. Sotalol was injected at minute 6 during 2 minutes.

2. Sinus rhythm conversion: A 50 minute recording obtained during an attempt to convert a patient with atrial fibrillation to sinus rhythm by using a series of head-up and head-down tilts.

3. Concatenated signals: In order to investigate the performance of the algorithm in a more complicated situation, eight 5 minute recordings were concatenated to one 40 minute recording. The different segments were diagnosed to have: 1) atrial fibrillation, 2) sinus rhythm, 3) atrial fibrillation with a ten seconds regular segment during the first minute, 4) sinus rhythm, 5) less organized atrial fibrillation, 6) sinus rhythm, 7) atrial flutter (pacemaker) and 8) atrial fibrillation.

\section{4. $\quad$ Results}

This section presents preliminary results from three examples, illustrating the potential of the present method. The signal characterization stage provides the present methods with one set of estimated parameters per second. Thus, the sampling rate of e.g. the frequency trend is 1 $\mathrm{Hz}$. In the subsequent analysis, the ARFT uses segments of $N_{1}=10$ samples and the trend detector is based on segments of $N_{2}=60$ samples, i.e., the feature tracker used blocks of ten seconds, and the detector blocks of 1 minute duration. The filter length and thresholds were empirically set to $N_{a}=20, \alpha=3, r_{1}=0.1, r_{2}=1.0, w_{1}=0.8$ and $w_{2}=1.2$.

The possibility to evaluate the effect of different interventions is illustrated by Fig. 1 where Sotalol is injected at minute 6 , decreasing the fibrillation frequency and causing the trend detector to search for a new stable frequency state. A few states are introduced before the frequency finally stabilizes due to that the difference in frequency during two consecutive segments is small. The entire signal is classified as an atrial tachyarrhythmia with irregular rate, mainly with a sawtooth-like shape (i.e., atrial fibrillation) except for a ten second segment where the signal is regular.

The second example illustrates the method's use in a cardiac monitoring system where sinus rhythm conversion is performed, see Fig. 2. A head-up tilt is performed at minute 15 with a duration of 5 minutes and a head-down tilt is performed at minute 30 with a duration of 10 minutes. Before conversion, the waveform is more pulse-shaped than sawtooth-like and is mainly irregular although with some

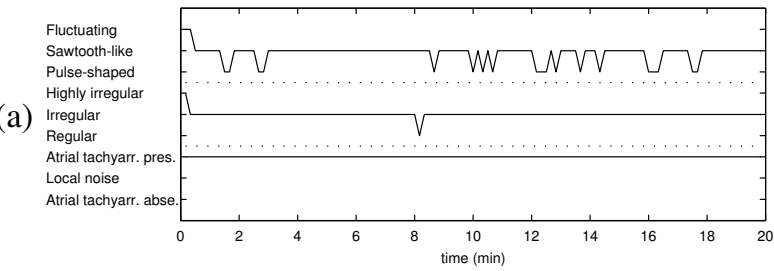

(b)

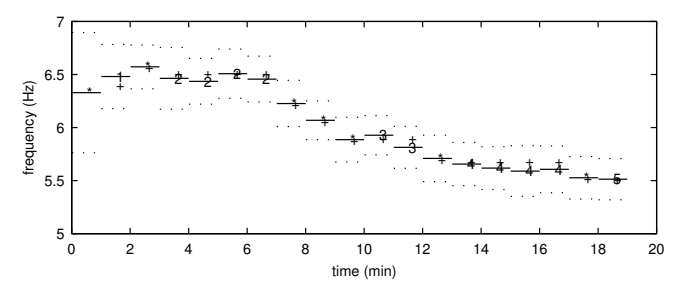

Figure 1. Sotalol intervention. (a) The three ARFT indicators. (b) The output from the FTD where the state frequencies $(+)$, the estimated mean frequencies (solid) and the standard deviations (dotted) for each segment are shown. The search state is indicated by $(*)$ and other states are indexed by $(1,2,3, \ldots)$.

intermittent intervals of a more regular rhythm. During the head-up tilt, some minor disturbances are detected and during the head-down tilt, the frequency, which starts at around $4.4 \mathrm{~Hz}$, decreases to a frequency around $4.25 \mathrm{~Hz}$ and then further to $4 \mathrm{~Hz}$. The atrial tachyarrhythmia disappears at minute 38 indicating a conversion to sinus rhythm.

Finally, the concatenated signals were analyzed: five seconds from each of the eight signal segments are shown in Fig. 3. In Fig. 4, the performance of the ARFT and FTD during the more complex transitions between the different rhythms are shown. Both the ARFT and the FTD successfully find the different rhythms and frequency states; the regular and more pulse-shaped flutter is detected, the three segments of sinus rhythm are detected and the segment with a more fluctuating high-frequency fibrillation is indicated differently than the slower and more sawtooth-shaped fibrillation. In addition, regular fibrillation interrupting the irregular fibrillation as well as intervals of local noise are found. It is noted that the algorithm falsely indicates an atrial tachyarrhythmia in two intervals during the second sinus rhythm segment being a result of residual $\mathrm{T}$ waves having the same order of magnitude as the $\mathrm{P}$ waves (see Fig. 3(f)).

\section{Discussion}

The purpose of the presented methods is to provide the physician with a more comprehensive description of the atrial signal. The reason for quantizing the rhythm features is the resulting ease of interpretation. The suggested frequency detector represents a simple approach 


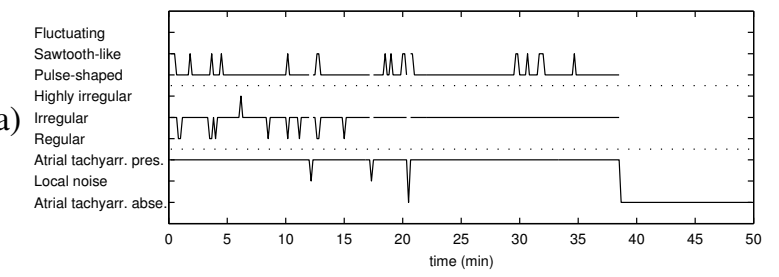

(b)

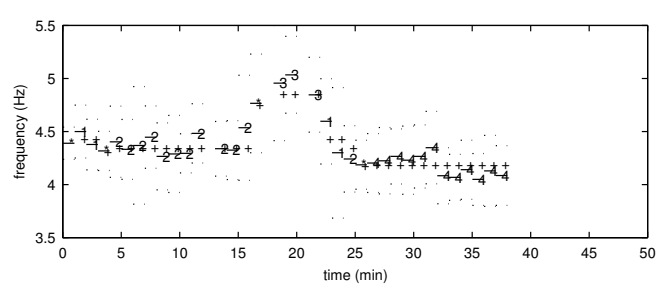

Figure 2. Sinus rhythm conversion. (a) The three ARFT indicators. (b) The output from the FTD where the state frequencies $(+)$, the estimated mean frequencies (solid) and the standard deviations (dotted) for each segment are shown. The search state is indicated by $(*)$ and other states are indexed by $(1,2,3, \ldots)$. (a)

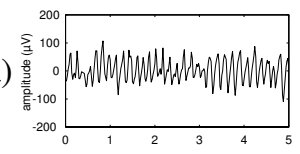

(b)

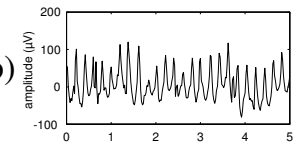

(c)
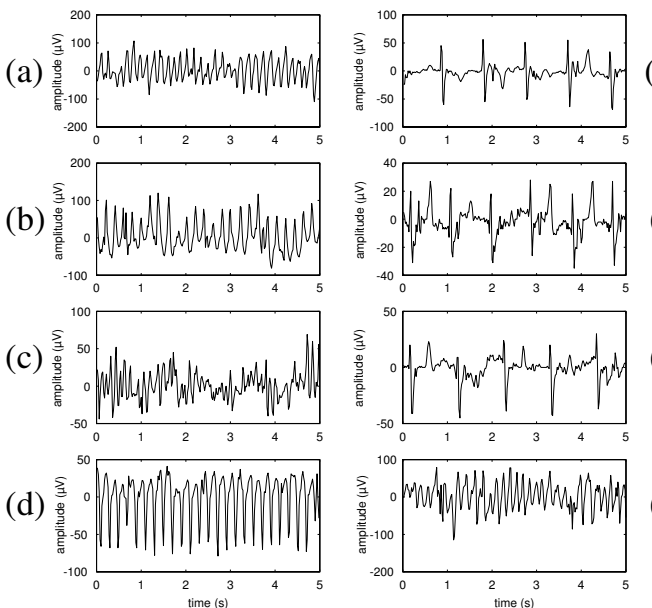

Figure 3. Concatenated signals. Five seconds from each of the eight signal segments containing (a), (b), (c) and (h) atrial fibrillation, (d) atrial flutter, and (e), (f) and (g) sinus rhythm.

to performing sequential trend detection. Other, more sophisticated methods for classification into frequency states can, of course, be used if the entire signal is available for analysis. More studies need to be done before the overall value of the method can be assessed.

\section{References}

[1] Stridh M, Sörnmo L, Meurling CJ, Olsson SB. Characterization of atrial fibrillation using the surface (a)

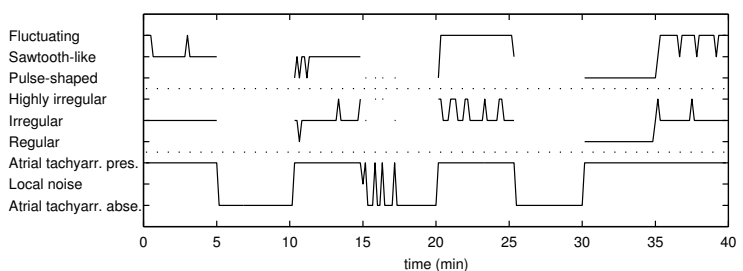

(b)

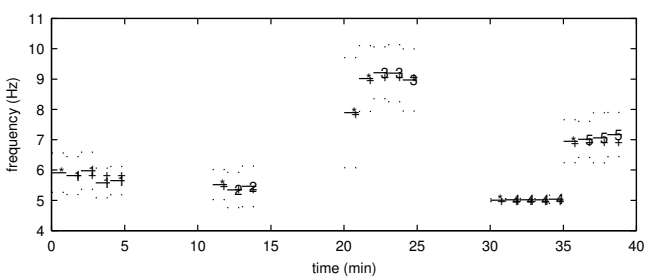

Figure 4. Concatenated signals. (a) The three ARFT indicators. (b) The output from the FTD where the state frequencies $(+)$, the estimated mean frequencies (solid) and the standard deviations (dotted) for each segment are shown. The search state is indicated by $(*)$ and other states are indexed by $(1,2,3, \ldots)$.

ECG: Time-dependent spectral properties. IEEE Trans Biomed Eng 2001;48(1):19-27.

[2] Langley P, Bourke JP, Murray A. Frequency analysis of atrial fibrillation. In Proc. Computers in Cardiology. IEEE Computer Society, 2000; 27:65-68.

[3] Rieta JJ, Zarzoso V, Millet Roig J, Garcia Civera R, Ruiz Granell R. Atrial activity extraction based on blind source separation as an alternative QRST cancellation for atrial fibrillation analysis. In Proc. Computers in Cardiology. IEEE Computer Society, 2000; 27:69-72.

[4] Stridh M, Sörnmo L. Spatiotemporal QRST cancellation techniques for analysis of atrial fibrillation. IEEE Trans Biomed Eng 2001;48(1):105-111.

[5] Stridh M, Sörnmo L, Meurling CJ, Olsson SB. Sequential characterization of atrial tachyarrhythmias based on ECG time-frequency analysis. IEEE Trans Biomed Eng 2003;(in press).

[6] $\mathrm{Ng} \mathrm{J}$, Sahakian A, Swiryn S. Vector analysis of atrial activity from surface ECGs recorded during atrial fibrillation. In Proc. Computers in Cardiology. IEEE Computer Society, 2002; 29:21-24.

Address for correspondence:

Martin Stridh

Dept. of Electroscience

Lund University

P.O. Box 118

SE-221 00 Lund

martin.stridh@es.lth.se 